\title{
EFECTO DEL DÉFICIT HÍDRICO DURANTE LA FASE JUVENIL SOBRE EL CRECIMIENTO Y ACUMULACIÓN DE PROLINA EN TRES GENOTIPOS DE PHASEOLUS VULGARIS L.
}

\section{EFFECT OF WATER DEFICIT DURING THE JUVENILE PHASE ON THE GROWTH AND PROLINE ACCUMULATION IN PHASEOLUS VULGARIS L. GENOTYPES}

Mailyn Mago, MSc. Magister Scientiarum en Agronomía (Venezuela). Profesora de la Facultad de Agronomía de la Universidad Central de Venezuela, Venezuela. mailynmagozam@gmail.com

Reinaldo Pire, Ph.D. Doctor en Producción Vegetal (Venezuela). Profesor de la Facultad de Agronomía Universidad de la Centroccidental Lisandro Alvarado, Venezuela. rpire@ucla.edu.ve Marina García, Ph.D. Doctora en Ciencias Agrícolas (Ecuador). Profesora de la Facultad de Ingeniería Agronómica de la Universidad Técnica de Manabí, Ecuador. marina.garcia@utm.edu.ec

\section{ARTÍCULO DE INVESTIGACIÓN}

Recibido: 4 de septiembre de 2020

Aceptado: 23 de diciembre de 2020

\section{RESUMEN}

Se investigó el efecto del déficit hídrico en tres genotipos de Phaseolus vulgaris L., con el objetivo de evaluar su impacto sobre el crecimiento y la acumulación foliar de prolina en fase juvenil. El ensayo se realizó en maceteros en condiciones de invernadero y los genotipos probados fueron Tacarigua, SA 018 y Selección 13. El déficit hídrico se impuso restringiendo el riego hasta alcanzar un potencial hídrico en el sustrato de $-0,08 \mathrm{MPa}$, dejando paralelamente un tratamiento control en el que el potencial hídrico se mantuvo en -0,01 MPa. A los 15 días después de iniciados los tratamientos, se cosecharon cuatro plantas por tratamiento y se determinaron las siguientes variables: área foliar, peso seco de la raíz y del vástago, relación raíz/vástago (R/V) y contenido foliar de prolina. En SA 018 el déficit hídrico redujo significativamente todas las variables de 
crecimiento, excepto la relación R/V, mientras que en Tacarigua y Selección 13, las mismas no se afectaron. La concentración foliar de prolina no varió significativamente con el déficit hídrico en ninguno de los genotipos. Estos resultados indican que Selección 13 y Tacarigua toleran el déficit hídrico en fase juvenil, mientras que SA 018 es sensible a este factor de estrés.

Palabras clave: crecimiento, prolina, déficit hídrico, Phaseolus vulgaris

\section{ABSTRACT}

The effect of the water deficit in three genotypes of Phaseolus vulgaris L. was investigated, in order to evaluate its impact on growth and foliar proline accumulation during the juvenile phase. The assay was made in pots under greenhouse conditions and the genotypes tested were Tacarigua, SA 018 and Selection 13. The water deficit was imposed by restricting irrigation until reaching a water potential in the substrate of $-0.08 \mathrm{MPa}$, leaving in parallel a control treatment in which the water potential remained at $-0.01 \mathrm{MPa}$. At 15 days after starting the treatments, four plants/treatment were harvested and the following variables were determined: Leaf area, root and shoot dry weight, root/shoot ratio (R/V) and foliar proline content. In SA 018, the water deficit significantly reduced all growth variables, except the R/V ratio, while in Tacarigua and Selection 13 , these variables were not affected. The foliar proline content did not vary significantly with the water deficit in none of the genotypes. These results indicate that Selection 13 and Tacarigua tolerate water deficit in the juvenile phase, while SA 018 is sensitive to this stress factor.

Key words: growth, proline, water deficit, Phaseolus vulgaris

\section{INTRODUCCIÓN}

El agua es un recurso natural de importancia vital para aumentar la producción de alimentos, lograr la seguridad alimentaria y mejorar las condiciones de vida de la sociedad (Rojas et al., 2020). La mayor parte de las tierras utilizadas para la agricultura requieren el uso de riego con agua dulce, sin embargo, el aumento de la población urbana, la industrialización creciente y el cambio climático, han hecho que el recurso hídrico sea cada vez más escaso para las actividades agrícolas (IICA, 2019).

En este contexto, el uso eficiente del agua para los cultivos se vislumbra como un aspecto clave para mejorar su productividad en condiciones de suministro hídrico restringido (IICA, 2017), poniendo de relieve la importancia de fortalecer la investigación dirigida a encontrar genotipos capaces de tolerar la sequía (Moreno 2009; Beekman, 2015; Rojas et al. 2020). 
Para lograr este objetivo, es fundamental conocer los mecanismos que utilizan los cultivos para enfrentar el déficit hídrico. En la planta, el estrés por deficiencia en el suministro hídrico es consecuencia de una disminución en el contenido de agua de los tejidos y ocurre cuando la tasa de transpiración excede a la tasa de absorción de agua por la raíz, lo que acarrea pérdida de turgencia, cierre de las estomas y una reducción en el crecimiento (Bathala, 2018).

Cuando la planta se enfrenta a condiciones de estrés hídrico, usualmente se afecta la expansión y el tamaño final de las hojas, con lo cual se reduce la tasa fotosintética pero la magnitud de este efecto depende de la intensidad y duración del período de escasez de agua, de la rapidez con la cual éste se alcance y además de la etapa fenológica en la cual se produzca (Chaves et. al. 2002; Bathala, 2018). Usualmente el crecimiento de la parte aérea se afecta más que el de las raíces y consecuentemente la relación raíz/vástago se incrementa, respecto a la de las plantas que no han sufrido sequía (Foxx \& Fort, 2019; Xu et al. 2015; Chaves et al. 2002), pero esta respuesta depende de la especie y también varía a nivel intraespecífico (Valladares \& Sánchez-Gómez, 2006).

Un mecanismo de gran importancia para superar el estrés hídrico es el ajuste osmótico, el cual se logra mediante la acumulación de solutos en las células, a fin de reducir el potencial hídrico, garantizando así el ingreso de agua a las mismas (Zivcak et al. 2016; Bathala, 2018). Entre los solutos que se asocian con el ajuste osmótico se encuentra la prolina, cuya función ha sido asociada con el mantenimiento de la turgencia de los tejidos y usualmente su contenido se incrementa bajo condiciones de estrés hídrico (Shakeri et al., 2019; Singh et al. 2017).

La concentración de prolina se ha utilizado como un sensor de distintos tipos de estrés abiótico (Singh et al., 2017) y en vista de la variación genotípica en la capacidad para acumular este aminoácido en respuesta al estrés hídrico, se ha sugerido su utilización como criterio de selección en programas de mejoramiento genético para tolerancia a la sequía en algunos cultivos (Heuer 2010; Dien et al., 2019). En el caso particular de $P$. vulgaris se ha sugerido una estrecha relación entre la capacidad para acumular este aminoácido y la sensibilidad de los genotipos ante la sequía (Arteaga et al., 2020).

Phaseolus vulgaris L. es una especie cuyo grano es rico en proteínas y en Venezuela, donde se le conoce con el nombre común de 'caraota', históricamente ha sido una de las leguminosas de mayor consumo cultivándose en todo el territorio nacional (Ochoa y Miranda, 2016), mayormente en pequeñas unidades de producción campesinas distribuidas en todas las regiones del país, por lo general con fines de autoconsumo y comercio local (De Gouveia et al., 2014). 
La siembra se efectúa básicamente en condiciones de secano, lo que genera cierta incertidumbre entre los agricultores ya que las plantas sólo disponen de la humedad almacenada en el suelo, y ello frecuentemente acarrea problemas de escasez de agua durante el ciclo del cultivo.

De acuerdo con los últimos datos disponibles en la literatura, el rendimiento promedio nacional de $P$. vulgaris en Venezuela se ubica alrededor de los $708 \mathrm{~kg} / \mathrm{ha}$ (FEDEAGRO, 2015), el cual se considera bajo tomando en cuenta que el potencial productivo de esta especie sobrepasa los $2000 \mathrm{~kg} / \mathrm{ha}$ (Sanders y Schwartz, 1980). Esta diferencia tan marcada, se atribuye a diversos factores, entre ellos la disponibilidad hídrica restringida durante el ciclo de desarrollo del cultivo (Pérez et al., 2018), ya que esta especie se considera sensible al déficit hídrico (Singh, 1995).

Una de las alternativas para aumentar la productividad de los materiales genéticos de $P$. vulgaris en condiciones de suministro hídrico restringido, es la selección de genotipos con características morfológicas y fisiológicas apropiadas para enfrentar la sequía (Montero-Tavera et al., 2019), para lo cual se requiere identificar estos caracteres a fin de que los mismos sean utilizados en programas de mejoramiento genético. El presente estudio tuvo como objetivo evaluar el efecto del déficit hídrico aplicado en la fase juvenil, sobre el crecimiento y la acumulación foliar de prolina en tres genotipos de $P$. vulgaris.

\section{MATERIALES Y MÉTODOS}

\section{Ubicación del ensayo}

La investigación fue realizada en un invernadero ubicado en el Instituto de Agronomía, Facultad de Agronomía de la Universidad Central de Venezuela en Maracay, estado Aragua Venezuela, ubicado a $10^{\circ} 17^{\prime} 03^{\prime \prime} \mathrm{LN}$ y $67^{\circ} 36^{\prime} 16^{\prime \prime}$ LO. La temperatura y humedad relativa promedio dentro del invernadero durante el lapso en el que se realizó el ensayo fue de $34,5 \stackrel{\circ}{\circ}$ y $67 \%$, respectivamente.

\section{Material vegetal}

Se utilizaron semillas de tres genotipos de Phaseolus vulgaris L.: SA 018, Selección 13 y la variedad Tacarigua, las cuales fueron suministradas por el Banco de Germoplasma del Instituto Nacional de Investigaciones Agrícolas - Centro Nacional de Investigaciones Agropecuarias (INIA/CENIAP) en Maracay estado Aragua, Venezuela. Los dos primeros genotipos, son materiales en proceso de mejoramiento genético que han mostrado buen comportamiento agronómico en Ensayos Regionales conducidos por este organismo en varias localidades del país (Información personal del Ing. Alberto Salih MSc., Mejorador del INIA/CENIAP). 
El genotipo Selección 13 ha tenido buen comportamiento en campo bajo condiciones de escaso suministro hídrico, mientras que el genotipo SA 018 ha tenido un comportamiento intermedio. Tacarigua es la variedad comercial más ampliamente utilizada por los agricultores en Venezuela (Ochoa \& Miranda, 2016; De Gouveia et al., 2014) y se considera sensible al déficit hídrico (Domínguez et al., 2014)

\section{Desinfección de las semillas y preparación de semilleros}

Antes de la siembra, las semillas se desinfectaron mediante inmersión en hipoclorito de sodio al 3\% durante un minuto y luego fueron sumergidas en una solución del fungicida Vitavax® (200 $\mathrm{cc} / 100 \mathrm{~kg}$ de semilla). Posteriormente, las semillas se sembraron en semilleros cuyo sustrato fue una mezcla de un suelo rico en materia orgánica y arena lavada de río en una proporción 3:1 a una profundidad de $2 \mathrm{~cm}$ aproximadamente y se regaron a diario con agua de grifo durante 10 días.

\section{Preparación del sustrato}

El sustrato que se utilizó fue una mezcla de un suelo rico en materia orgánica y arena lavada de río en proporción 3:1, la cual se esterilizó mediante la aplicación de vapor por una hora, se dejó secar y luego se colocó en recipientes plásticos de $17 \mathrm{~cm}$ de diámetro y $14,5 \mathrm{~cm}$ de altura a razón de 2,1 kg/recipiente, ejerciendo cierta presión al dejarlo caer desde poca altura, a fin de asentar el material sin llegar a compactarlo y luego se aplicó un primer riego con abundante agua hasta saturar el sustrato y se dejó que los recipientes drenaran completamente. Una muestra de esta mezcla fue utilizada para la obtención de una curva de calibración utilizando un tensiómetro y una balanza, para conocer la tensión que existía en el sustrato en cualquier momento mediante la obtención de su peso.

\section{Trasplante e inicio de los tratamientos}

Transcurridos 10 días después de la siembra, se seleccionaron 12 plantas lo más homogéneas posible de cada genotipo a partir de los semilleros, se trasplantaron a las macetas y se iniciaron los riegos diarios reponiendo el agua faltante para mantener la tensión de humedad del sustrato a capacidad de campo (CC), lo cual correspondió a un potencial de -0,01 MPa. Diez días después del trasplante, las plantas de cada genotipo se separaron en dos lotes, en uno de ellos se suspendió el riego y solo se repuso el agua faltante cuando el potencial hídrico del sustrato alcanzó un valor de -0,08 MPa (tratamiento de déficit hídrico), mientras que en la otra mitad de las plantas se mantuvo el potencial hídrico en -0,01 MPa (tratamiento control). Estos tratamientos se mantuvieron durante 15 días. 


\section{Diseño experimental}

Se empleó un diseño experimental completamente aleatorizado, en un arreglo de tratamientos factorial $3 \times 2$, es decir, tres genotipos dos regímenes de humedad y cuatro repeticiones por tratamiento.

\section{Variables evaluadas}

Finalizado el experimento ( 25 días después del trasplante), se cosecharon cuatro plantas por tratamiento de manera aleatoria y se realizaron las siguientes evaluaciones:

- Área foliar

Se determinó el área foliar de todas las hojas de cada planta seleccionada utilizando un medidor de área foliar marca CID-Inc. modelo Cl-202.

- $\quad$ Peso seco de la raíz, peso seco del vástago y relación raíz/vástago (R/V)

En cada planta muestreada se separó la parte aérea del sistema radical. En el caso de las raíces éstas se lavaron con agua y un cedazo para evitar pérdidas de material radical y así eliminar restos de sustrato, posteriormente se secaron con papel absorbente; el material vegetal correspondiente a la parte aérea y a las raíces y se llevó a estufa a $70 \stackrel{\circ}{C}$ por 72 horas y se registró el peso seco de cada componente; con esos valores se calculó la relación peso seco de raíz/peso seco vástago $(\mathrm{R} / \mathrm{V})$.

- Contenido foliar de prolina

El contenido de prolina se determinó en muestras secas correspondientes a la primera hoja trifoliada de cuatro plantas por tratamiento, las cuales se secaron en una estufa ventilada a 70 ${ }^{\circ} \mathrm{C}$ por $48 \mathrm{~h}$ y el material seco se molió en un molino eléctrico marca Arthur Thomas con malla número 60.

Para determinar el contenido de prolina, se usó la metodología propuesta por Bates et al. (1973), basada en la formación de un complejo coloreado cuya absorbancia se midió en un espectrofotómetro modelo 1100 RS marca Milton Ray a $520 \mathrm{~nm}$. Previamente se elaboró una curva patrón utilizando L- prolina SigmaUltra ${ }^{\circledR}$. El contenido de prolina en las muestras se obtuvo mediante la siguiente relación:

$\mu \mathrm{mol}$ prolina/g peso seco = (Concentración* ml tolueno/ 115,5 $\mu \mathrm{g} / \mu \mathrm{mol}) /(\mathrm{g}$ muestra/ 5)

\section{Análisis estadístico de los resultados}

Los resultados correspondientes a las variables determinadas en este ensayo se organizaron en bases de datos y se realizó un análisis de varianza (ANOVA) de acuerdo al diseño propuesto. 
En el caso de aquellas variables que resultaron con diferencias significativas entre los tratamientos, se realizó la prueba de comparación de medias de Tukey. Los análisis estadísticos se efectuaron con la ayuda del programa Statistix para Windows versión 8.0.

\section{RESULTADOS Y DISCUSIÓN}

En la Tabla 1 se muestran los valores de probabilidad (p), obtenidos mediante del análisis de varianza (ANAVAR) para genotipo, tratamiento y la interacción tratamiento $x$ genotipo, en las variables determinadas en los tres genotipos de $P$. vulgaris sometidos a déficit hídrico y seguidamente se describe el comportamiento de cada una de ellas.

Tabla 1

Valores de probabilidad (p) para genotipo, tratamiento e interacción obtenidos mediante el análisis de varianza (ANAVAR) para las variables determinadas en tres genotipos de $P$. vulgaris sometidos a déficit hídrico.

\begin{tabular}{lccc}
\hline \multicolumn{1}{c}{ Variable } & Genotipo(G) & Tratamiento(T) & GxT \\
\hline Área foliar & 0,0962 & 0,0002 & 0,0340 \\
Peso seco raíz & 0,0919 & 0,1660 & 0,0211 \\
Peso seco vástago & 0,0297 & 0,1448 & 0,0000 \\
Relación raíz/vástago & 0,0000 & 0,2117 & 0,7905 \\
Prolina & 0,0000 & 0,0323 & 0,5553 \\
\hline
\end{tabular}

Nota. GxT: interacción genotipo x tratamiento. Fuente: Elaboración propia

\section{Área foliar}

En cuanto a la variable área foliar, no se detectaron diferencias significativas entre los genotipos (Tabla 1), mientras que para tratamientos si hubo diferencia $(p \leq 0,01)$ e igualmente para la interacción genotipo $x$ tratamiento $(p \leq 0,05)$.

En el tratamiento control la mayor área foliar correspondió a SA 018, seguido por Selección 13 y Tacarigua (Figura 1). El déficit hídrico provocó una reducción en el área foliar en los tres genotipos, pero la proporción de la misma fue distinta en cada uno de ellos, alcanzando 13\%, $19 \%$ y $46 \%$ respecto al control, en Selección 13, Tacarigua y SA 018 respectivamente, lo cual evidencia la alta sensibilidad de este último genotipo ante el déficit hídrico impuesto. 


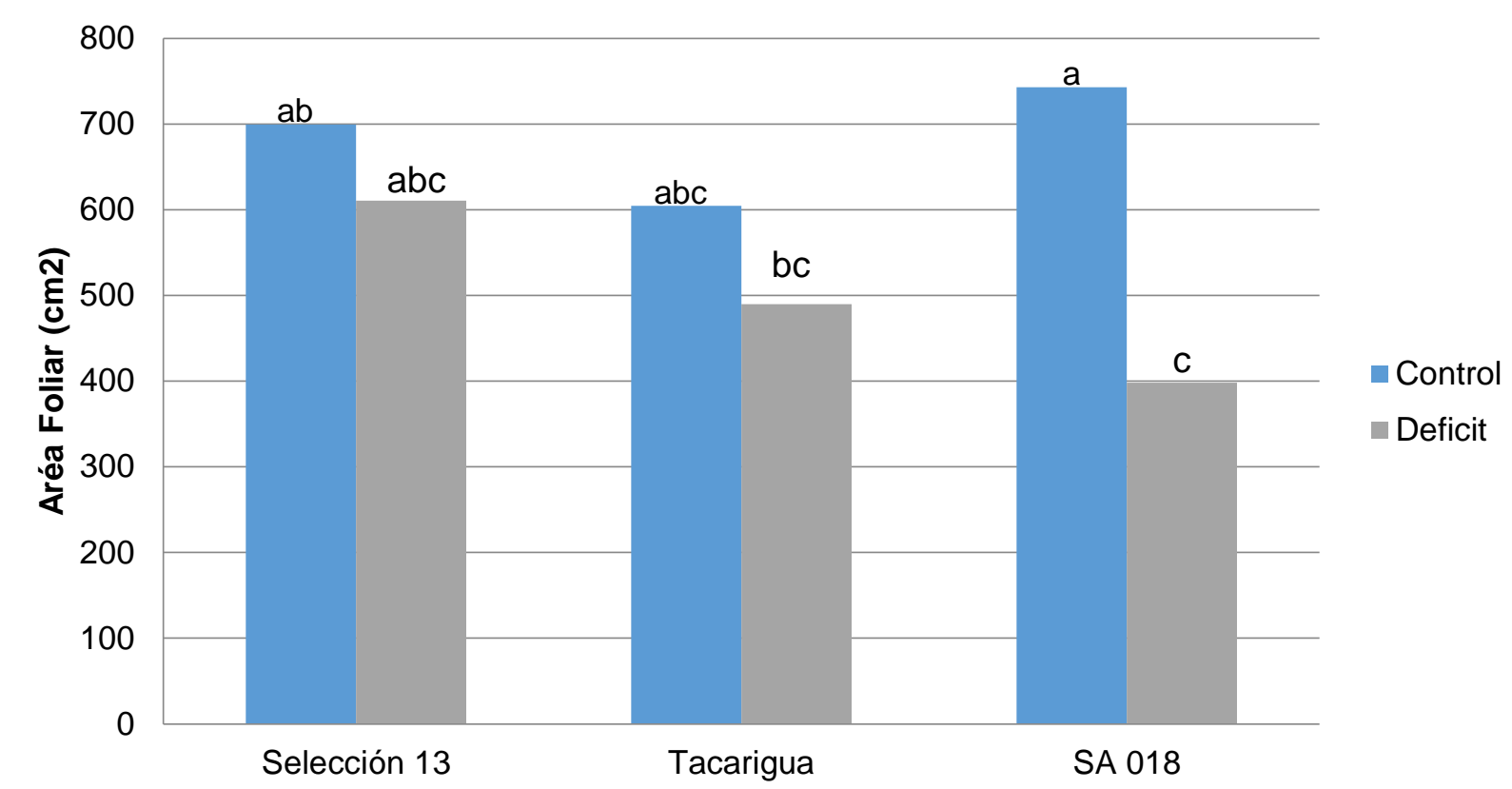

Figura 1. Valores promedio de área foliar en los tres genotipos de $P$. vulgaris creciendo en condiciones de suplencia adecuada de agua (control) o bajo déficit hídrico.

Nota. Letras distintas sobre las barras indican diferencia significativa para la interacción $(p \leq 0,05)$ según la prueba de medias de Tukey. Fuente: Elaboración propia

En forma similar a lo observado en este estudio, trabajos previos han demostrado un acentuado impacto detrimental de la sequía sobre el área foliar en $P$. vulgaris (Kalaydjieva et al., 2015; Reyes-Matamoros et al., 2014).

\section{Peso seco de la raíz y del vástago}

Para la variable peso seco de la raíz no se detectó diferencia significativa $(p \leq 0,05)$ para ninguno de los dos factores bajo estudio, pero si para la interacción genotipo $(G) \times$ tratamiento (T) (Tabla 1).

En el genotipo SA 018 la condición de déficit hídrico provocó una reducción significativa en la biomasa de raíces cuya magnitud fue de $29 \%$ con respecto al tratamiento control, mientras que en la variedad Tacarigua y el genotipo Selección 13 la acumulación de biomasa en el sistema radical fue similar en las dos condiciones de suministro hídrico (Figura 2). 


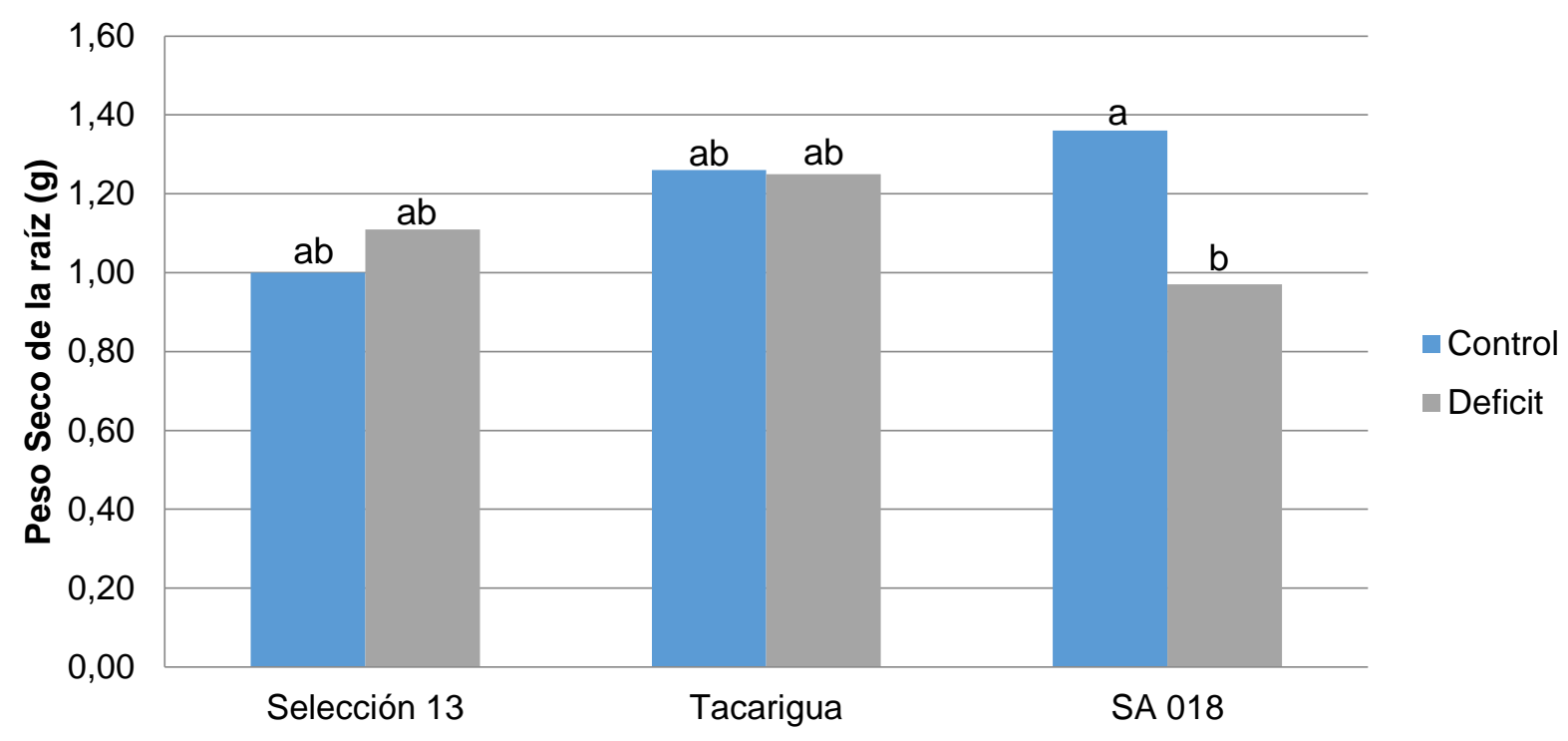

Figura 2. Valores promedio del peso seco de la raíz ( $\mathrm{g}$ ) en tres genotipos de P. vulgaris creciendo en condiciones de suplencia adecuada de agua (control) o bajo déficit hídrico.

Nota. Letras distintas sobre las barras indican diferencia significativa para la interacción $(p \leq 0,05)$ según la prueba de medias de Tukey. Fuente: Elaboración propia

En cuanto a la variable peso seco de la parte aérea o vástago, se observó diferencia significativa $(p \leq 0,05)$ para genotipo y para la interacción genotipo $x$ tratamiento (Tabla 1$)$ y en forma similar a lo observado en la raíz, el déficit hídrico solo provocó una reducción significativa en el peso seco del vástago en el genotipo SA 018 donde esta variable disminuyó en un 33\% respecto al tratamiento control, mientras que en la variedad Tacarigua y en el genotipo Selección 13 esta variable no resultó afectada (Figura 3).

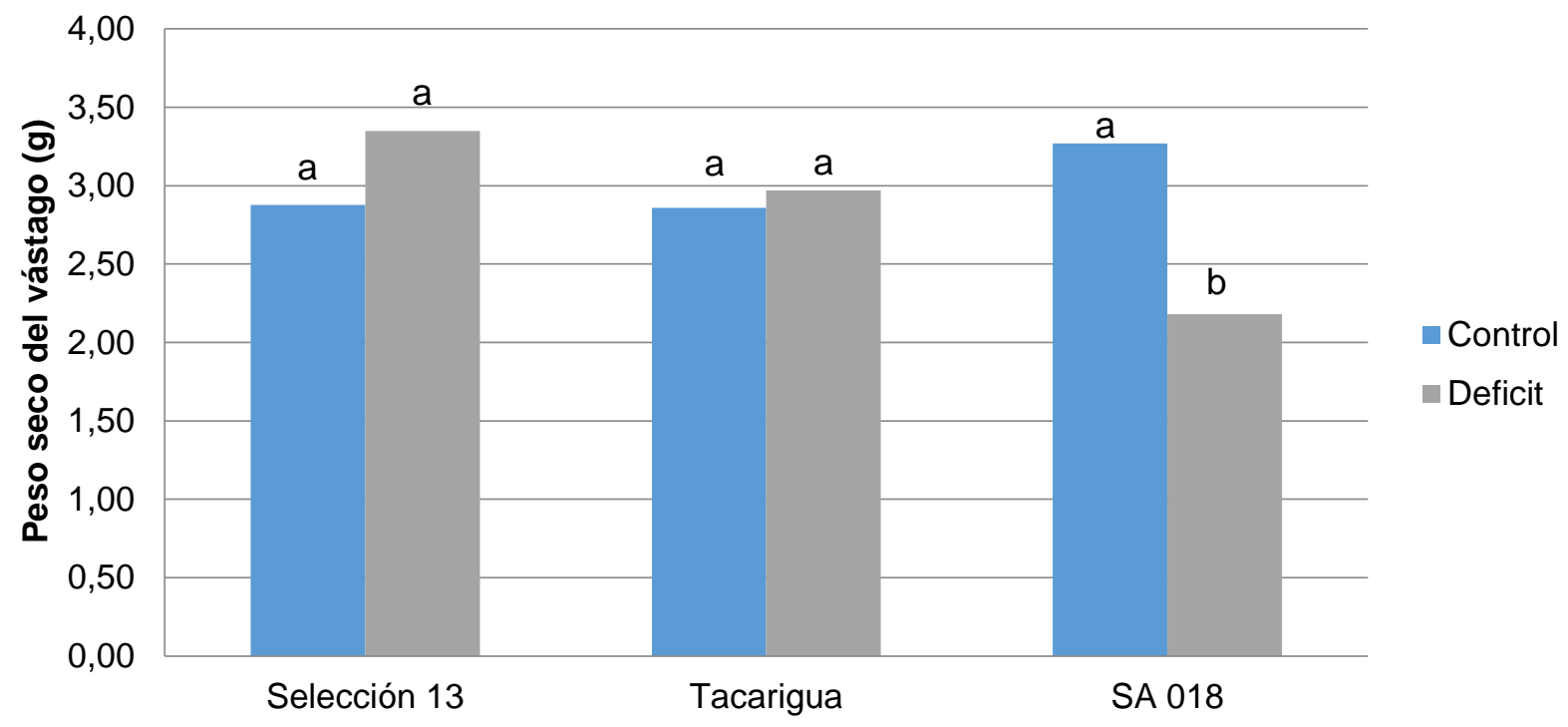

Figura 3. Valores promedio del peso seco del vástago en los tres genotipos de P. vulgaris creciendo en condiciones de suplencia adecuada de agua (control) o bajo déficit hídrico.

Nota. Letras distintas sobre las barras indican diferencia significativa para la interacción $(p \leq 0,05)$ según la prueba de medias de Tukey. Fuente: Elaboración propia 
El déficit hídrico solo afectó la acumulación de biomasa radical y aérea en el genotipo SA 018, siendo ese efecto ligeramente mayor en el vástago que en la raíz, lo que es indicativo de la sensibilidad de este genotipo al estrés hídrico impuesto. Por el contrario, en el genotipo Selección 13 y en la variedad Tacarigua el déficit hídrico aplicado no tuvo efecto sobre la biomasa acumulada en esos dos componentes de la planta, lo cual evidencia que en los mismos se comportaron como tolerantes al déficit hídrico.

En el caso de la variedad Tacarigua, ese hallazgo contrasta con lo señalado por De Gouveia et al. (2014), quienes indicaron que la misma es sensible al déficit hídrico, no obstante, es oportuno puntualizar que estos investigadores trabajaron a nivel de campo y observaron el comportamiento de la variedad Tacarigua en la etapa reproductiva, la cual ha sido señalada como la más sensible a la sequía en $P$. vulgaris (Calvacache et al. 1997).

Otro aspecto que cabe destacar, es que estos resultados demuestran que el área foliar se redujo en mayor magnitud que la acumulación de biomasa en la parte aérea, lo cual coincide con lo reportado por otros autores quienes han indicado que este es uno de los parámetros más afectados por la escasez de agua (Bathala, 2018; Hussain \& Ali, 2015; Moreno, 2009).

\section{Relación raíz/vástago (R/V)}

Con respecto a la relación $R / V$, solo se detectó diferencia significativa $(p \leq 0,05)$ para genotipo (Tabla 1). En las plantas del tratamiento control la relación R/V fue notablemente menor en el genotipo Selección 13, con respecto a los otros dos genotipos estudiados en los cuales la misma fue similar (Figura 4).

Bajo la condición de suministro hídrico restringido, la relación raíz/vástago se incrementó en $9 \%, 7 \%$ y $1 \%$ respecto al tratamiento control, en los genotipos Selección 13, SA 018 y variedad Tacarigua respectivamente, lo que evidencia que solo en los dos primeros genotipos el déficit hídrico provocó un ligero incrementó en la proporción de asimilados invertidos en producir biomasa de raíz a expensas de la biomasa aérea. 


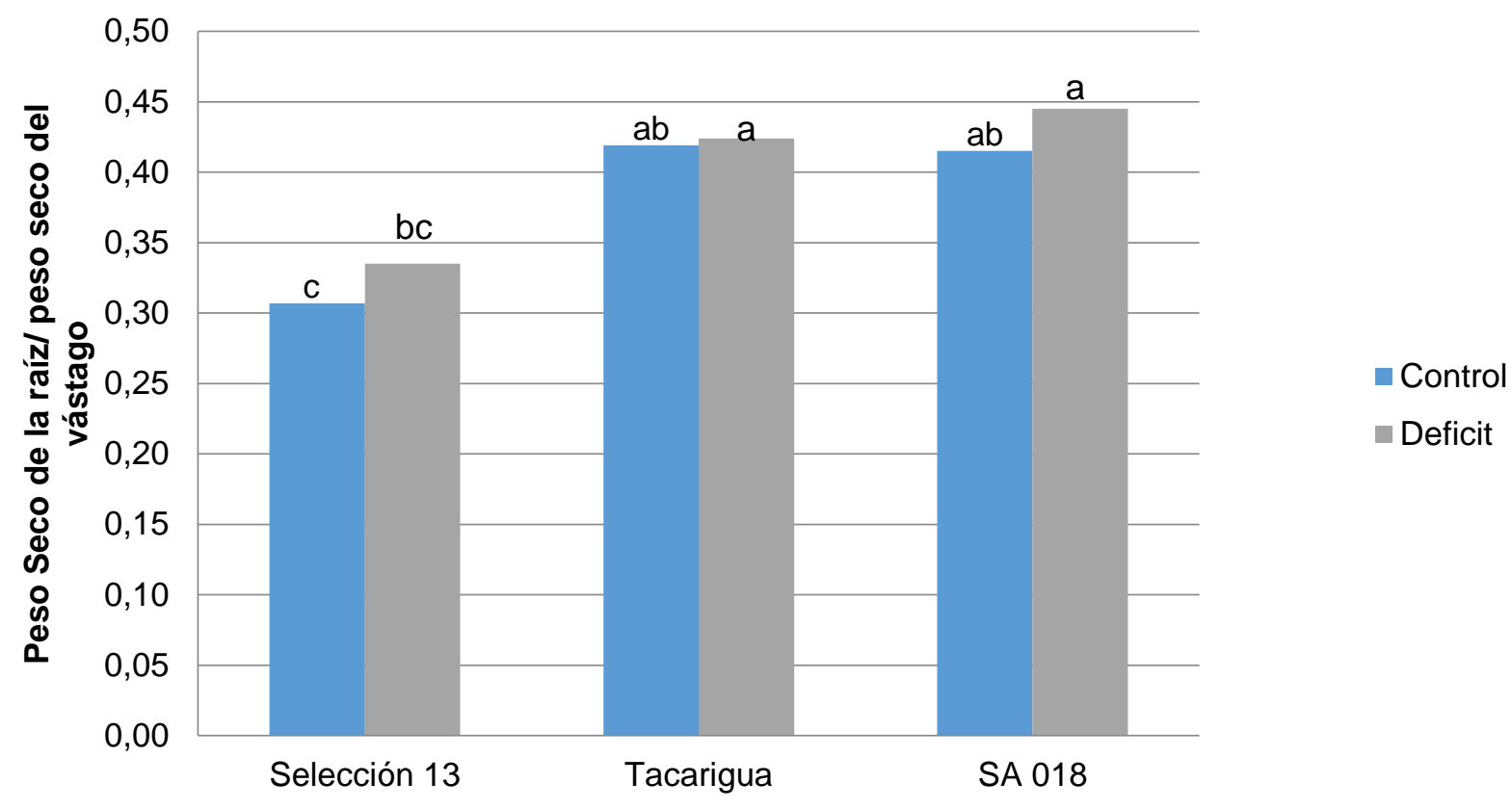

Figura 4. Valores promedio de la relación peso seco de la raíz/peso seco del vástago, en los tres genotipos de P. vulgaris creciendo en condiciones de suplencia adecuada de agua (control) o bajo déficit hídrico.

Nota. Letras distintas sobre las barras indican diferencia significativa para la interacción $(p \leq 0,05)$ según la prueba de medias de Tukey. Fuente: Elaboración propia

En condiciones de baja disponibilidad hídrica las estomas tienden a cerrar para limitar la pérdida de agua, con lo cual se reduce la captura de $\mathrm{CO} 2$ y disminuye la tasa de fotosíntesis (Bhatla, 2018; Montero-Tavera et al., 2019).

Bajo estas circunstancias, la planta puede responder transportando más foto asimilados al sistema radical para promover un mayor desarrollo del mismo y favorecer de esta manera la absorción de agua (Foxx y Fort, 2019), con lo cual la relación R/V tiende a incrementarse (Moreno, 2009; Xu et al., 2015).

De acuerdo con los resultados obtenidos en esta investigación, el genotipo Selección 13 tiene la potencialidad genética para desarrollar una mayor relación $\mathrm{R} / \mathrm{V}$, lo cual puede considerarse una ventaja para enfrentar condiciones de sequía, y aunque para esta relación no se detectaron diferencias significativas entre tratamientos, si se observó una tendencia a un incremento en la misma, especialmente en el genotipo Selección 13.

Lo cual supone una mayor inversión de asimilados para el crecimiento del sistema radical bajo la condición de restricción en el suministro de agua, lo que coincide con lo observado por Montero-Tavera et al. (2019) en genotipos de frijol (P. vulgaris). 


\section{Acumulación foliar de prolina}

La concentración foliar de prolina resultó con diferencias significativas para genotipos (G) y tratamientos $(T)(p \leq 0,05)$, no así para la interacción GxT (Tabla 1).

En el tratamiento control, la variedad Tacarigua mostró la mayor concentración foliar de prolina, mientras que en los genotipos Selección 13 y SA 018 ésta fue sustancialmente menor y similar en ambos (Figura 5).

La condición de déficit hídrico provocó un aumento en la concentración de prolina en la hoja que fue de 1,7, 1,3 y 1,2 veces, respecto al tratamiento control, en el genotipo Selección 13, variedad Tacarigua y genotipo SA 018 respectivamente, y aunque las medias fueron similares para las dos condiciones, es claro que en el primer genotipo la magnitud del incremento en la concentración de este aminoácido por efecto del déficit hídrico fue mayor.

Es de hacer notar que en el tratamiento control la acumulación foliar de prolina en la variedad Tacarigua, fue superior a la de los otros dos genotipos, lo que refleja que la capacidad para acumular este aminoácido es una condición intrínseca de esta variedad.

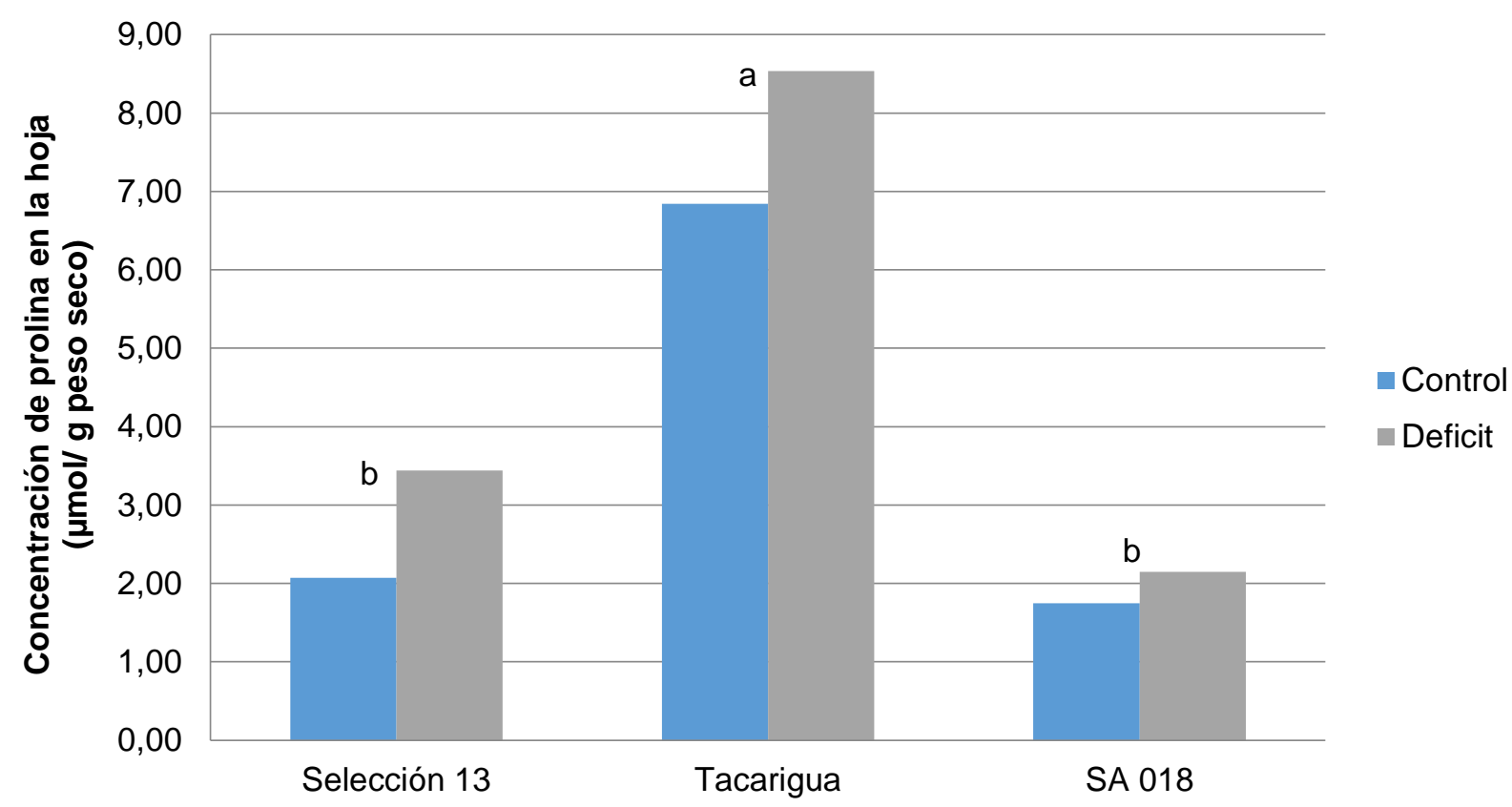

Figura 5. Valores promedio de concentración de prolina en la hoja ( $\mu \mathrm{mol} / \mathrm{g}$ peso seco) en tres genotipos de P. vulgaris creciendo en condiciones de suplencia adecuada de agua (control) o bajo déficit hídrico.

Nota. Letras distintas sobre las barras indican diferencia significativa para la interacción $(p \leq 0,05)$ según la prueba de medias de Tukey. Fuente: Elaboración propia 
Estos resultados coinciden con lo reportado por otros investigadores, quienes han señalado que muchas especies tienden a incrementar la producción de prolina como respuesta rápida a la sequía, lo cual las ayuda a mantener el ajuste osmótico sin alterar su metabolismo (Shakeri et al, 2019; Singh et al., 2017; Zivcak et al., 2016). En el caso particular de P. vulgaris se ha considerado que el comportamiento en cuanto a la acumulación de prolina en plantas bajo déficit hídrico puede ser usado como un indicador que refleja el estado hídrico de la planta, ya que se ha encontrado que el contenido de este aminoácido aumenta más en los genotipos sensibles, en comparación con los tolerantes (Arteaga et al., 2020; Domínguez et al., 2014), lo cual contrasta con el comportamiento observado en este estudio durante la fase juvenil de este cultivo.

La respuesta de tolerancia de las plantas a la sequía, implica la intervención de una serie de mecanismos morfológicos, anatómicos, fisiológicos, bioquímicos y moleculares (Domínguez et al., 2014) y la identificación de marcadores asociados a cada uno de ellos resulta una herramienta muy valiosa para la selección de genotipos tolerantes. El análisis de las variables determinadas en esta investigación, muestra que el genotipo Selección 13 y la variedad Tacarigua toleraron el déficit hídrico impuesto, mientras que el genotipo SA 018 claramente mostró ser bastante sensible a la restricción en el suministro hídrico. No obstante, este estudio solo abarcó parte de la fase juvenil, y se ha indicado que en $P$. vulgaris la etapa reproductiva es la más sensible a la sequía (Calvacache et al. 1997).

\section{CONCLUSIONES}

El genotipo SA 018 resultó sensible al estrés por déficit hídrico, mientras que el genotipo Selección 13 y la variedad Tacarigua se comportaron como tolerantes a esta condición, al menos para el nivel y duración del déficit hídrico impuesto durante la fase juvenil.

\section{REFERENCIAS BIBLIOGRÁFICAS}

Arteaga, S., Yabor, L., Díez, M., Prohens, J., Boscaiu, M., y Vicente, O. (2020). The Use of Proline in Screening for Tolerance to Drought and Salinity in Common Bean (Phaseolus vulgaris L.) Genotypes. Agronomy, 10(6), 817. Recuperado de https://www.mdpi.com/2073-4395/10/6/817/pdf

Bates, L., Waldren, R., y Teare, D. (1973). Rapid determination of free proline for water-stress studies. Plant Soil, 39, 205-207. Recuperado de https://link.springer.com/content/pdf/10.1007/BF00018060.pdf

Beekman, G. (2015). Agua y seguridad alimentaria. [Archivo PDF]. Recuperado de http://scioteca.caf.com/handle/123456789/784 
Bhatla, S. (2018). Abiotic Stress. En: Plant Physiology, Development and Metabolism (pp. 9691028). Singapore:Springer.

Calvacache, M., Reichard, K., Bacchp, O., y Dourado-Neto, N. (1997). Deficit irrigation at different growth stages of the common bean (Phaseolus vulgaris L., cv. Imbabello). Sci. agric. (Piracicaba, Braz.), $54 \quad(1-16), \quad$ Recuperado de https://www.scielo.br/scielo.php?script=sci_arttext\&pid=S0103-

$90161997000300002 \&$ Ing $=$ pt\&nrm=iso

Confederación de Asociaciones de Productores Agropecuarios de Venezuela (FEDEAGRO). (2015). Estadísticas Agrícolas. Disponible en https://fedeagro.org/estadisticasagricolas/produccion-agropecuaria/produccion/

Chaves, M., Pereira, J., Maroco, J., Rodrigues, M., Ricardo, C., Osório, M., Carvalho, I., Faria, T., y Pinheiro, C. (2002). How plants cope with water stress in the field. Photosynthesis and growth. Annals of Botany, 89 (7), 907-916. Recuperado de https://academic.oup.com/aob/article/89/7/907/151103

De Gouveia, M., Gámez, A., y Pérez, H. (2014). Evaluación y selección participativa de cultivares de caraota. Agronomía Tropical, 64(1-2), 49-59. Recuperado de http://ve.scielo.org/scielo.php?script=sci_arttext\&pid=S0002-192X2014000100006

Dien, D., Mochizuki, T., y Yamakawa, T. (2019). Effect of various drought stresses and subsequent recovery on proline, total soluble sugar and starch metabolisms in Rice (Oryza sativa L.) varieties. Plant Production Science, 23 (4), 355-365. Recuperado de https://www.tandfonline.com/doi/full/10.1080/1343943X.2019.1647787

Domínguez, A., Pérez, Y., Alemán, S., Sosa, M., Fuentes L., Darias, R., Demey, J., Rea, R., y Sosa, D. (2014). Respuesta de cultivares de Phaseolus vulgaris L. al estrés por sequía. Biotecnología Vegetal, 14(1), Recuperado de https://revista.ibp.co.cu/index.php/BV/article/view/38/432

Foxx A., y Fort, F. (2019) Root and shoot competition lead to contrasting competitive outcomes under water stress: A systematic review and meta-analysis. PLOS ONE 14(12). Recuperado de https://journals.plos.org/plosone/article?id=10.1371/journal.pone.0220674

Heuer B (2010) Role of proline in plant response to drought and salinity. In: Pessarakli M (ed) Handbook of plant and crop stress. CRC Press, Boca Raton, pp 213-238

Hussain, B., y Ali, B. (2015). Leaf longevity in plants under water stress - A Review. Indian Journal of Plant Sciences, 4(4), 127-133. Recuperado de https://www.researchgate.net/publication/313506661_LEAF_LONGEVITY_IN_PLANTS_ UNDER_WATER_STRESS_-_A_REVIEW 
Instituto Interamericano de Cooperación para la Agricultura (IICA). (2019). Perspectivas de la agricultura y del desarrollo rural en las Américas: una mirada hacia América Latina y el Caribe 2019-2020.

Disponible

en https://repositorio.iica.int/bitstream/handle/11324/8214/BVE19040295e.pdf?sequence=1

Instituto Interamericano de Cooperación para la Agricultura (IICA). (2017). El agua para la agricultura de las Américas. Disponible en https://repositorio.iica.int/bitstream/handle/11324/6148/BVE17109367e.pdf?sequence=1

Kalaydjieva, R., Matey, A., y Zlatev, Z. (2015). Influence of irrigation regime on the leaf area and leaf area index of French bean (Phaseolus vulgaris L.). Emir. J. Food Agric. 27 (2): 171 177. Recuperado de https://www.researchgate.net/publication/273289149_Influence_of_irrigation_regime_on _the_leaf_area_and_leaf_area_index_of_French_bean_Phaseolus_vulgaris_L

Montero, V., Gutiérrez, G., Mireles, A., Aguirre, C., Acosta, J., y Ruiz, J. (2019) Efectos fisiológicos del estrés hídrico en variedades de frijol tolerantes a sequía. Acta Universitaria, 29, 1-7. Recuperado de http://www.scielo.org.mx/scielo.php?pid=S018862662019000100114\&script=sci_arttext

Moreno, L. (2009). Respuesta de las plantas al estrés por déficit hídrico. Una revisión. Agronomía Colombiana, $27 \quad$ (2), 179-191. Recuperado de https://revistas.unal.edu.co/index.php/agrocol/article/download/11131/37762

Ochoa, E., y Miranda, A. (2016). Somos de caraotas [Archivo PDF]. Recuperado de http://biblioteca.clacso.edu.ar/clacso/se/20191105044227/Somos_de_Caraota.pdf

Pérez, H., De Gouveia, M., Viera, F., y Méndez. A. (2018). Evaluación de cultivares de caraota (Phaseolus vulgaris, L.) en zona de colinas del estado Guárico, Venezuela. Ojeando la Agenda, 57. Recuperado de https://ojeandolaagenda.com/2019/01/31/evaluacion-decultivares-de-caraota-phaseolus-vulgaris-I-en-zona-de-colinas-del-estado-guaricovenezuela/

Reyes, J., Martínez, D., Rueda, R., y Rodríguez, T. (2014). Efecto del estrés hídrico en plantas de frijol (Phaseolus vulgaris L.) en condiciones de invernadero. Revista Iberoamericana de Ciencias, 1(2), 191-203. Recuperado de http://www.reibci.org/publicados/2014/julio/2200132.pdf

Rojas, F., Peñaherrera, F., Orellana, C., Castañeda, H., Armijos, L., Burbano, L., Morales, A., Rodrigues, P., Real, C., Rispo, A., Valverde, O., Alonso, A., y Bianchi, F. (2020). Water Strategy 2019-2022. [Archivo PDF] Recuperado de https://scioteca.caf.com/bitstream/handle/123456789/1578/Water_Strategy_20192022.pdf?sequence $=1$ \&isAllowed $=y$ 
Sanders, J., Schwartz, H. (1980). Bean production and pest constraints in Latin America. [Archivo PDF] Recuperado de http://ciatlibrary.ciat.cgiar.org/Articulos_Ciat/biblioteca/Bean_Production_Problems_in_the_Tropic s.pdf

Shakeri, E., Mozafari, A., Sohrabi, F. y Saed-Moucheshi, A. (2019). Role of Proline and Other Osmoregulatory Compounds in Plant Responses to Abiotic Stresses. En: Pessarakli, M. (eds), Handbook of Plant and Crop Stress, (pp: 974-983) Boca Raton: CRC Press.

Singh, A., Kumar M., y Sengar. R. (2017). Osmolytes: Proline metabolism in plants as sensors of abiotic stress. Journal of Applied and Natural Science, 9 (4), 2079 -2092. Recuperado de https://core.ac.uk/download/pdf/158353677.pdf

Singh, S. (1995). Selection for water stress tolerance in interracial populations of common bean. Crop Science, 35: 118-124. Recuperado de https://acsess.onlinelibrary.wiley.com/doi/abs/10.2135/cropsci1995.0011183X00350001 0022x

Torres, E., Quisphe, D., Sánchez, A., Reyes, M., González, B., Torres, A., Cedeño, A., y Haro, A. (2013). Caracterización de la producción de frijol en la provincia de Cotopaxi Ecuador: Caso Comuna Panyatug. Ciencia y Tecnología, 6(1), 23-31. Recuperado de https://www.researchgate.net/publication/295260066_CARACTERIZACION_DE_LA_PR ODUCCION_DE_FRIJOL_EN_LA_PROVINCIA_DE_COTOPAXI_ECUADOR_CASO_C OMUNA_PANYATUG

Valladares, F., y Sánchez, D. (2006). Ecophysiological Traits Associated with Drought in Mediterranean Tree Seedlings: Individual Responses versus Interspecific Trends in Eleven Species. Plant Biol, 8: 688 - 697. Recuperado de https://doi.org/10.1055/s-2006924107

Xu W., Cui, K., Xu, A., Nie, L., Huang, L., y Peng. S. (2015). Drought stress condition increases root to shoot ratio via alteration of carbohydrate partitioning and enzymatic activity in rice seedling. Acta Physiology Plant, 37:9 Recuperado de https://doi.org/10.1007/s11738-0141760-0

Zivcak M., Brestic M., y Sytar O. (2016) Osmotic Adjustment and Plant Adaptation to Drought Stress. En: Hossain M., Wani S., Bhattacharjee S., Burritt D., Tran LS. (eds), Drought Stress Tolerance in Plants. 1. (pp.105-143). India: Springer 